\title{
LA EVALUACIÓN EN EDUCACIÓN FÍSICA Revisión de modelos tradicionales y planteamiento de una alternativa. La evaluación formativa y compartida
}

\section{Autores}

Víctor M. López Pastor (coordinador), Roberto Monjas Aguado, Jesús Gómez García, Esther M. López Pastor, Juan F. Martín Pinela, Javier González Badiola, José Juan Barba Martín, Rebeca Aguilar Baeza, Marta González Pascual, Carlos Heras Bernardino, Maria Isabel Martín, Juan Carlos Manrique Arribas, Patricia Subtil Marugán, Laura Marugán García

Grupo de trabajo internivelar de investigación-acción en Educación Física de Segovia. (E. U. Magisterio de Segovia - Centro Buendía de la Universidad de Valladolid)

\section{Resumen}

Este artículo está organizado en tres partes. En la primera realizamos un análisis crítico de los modelos tradicionales de evaluación-calificación en Educación Física, de un modo muy sintético. Son diez buenas razones para intentar superarlo lo antes posible. En la segunda parte analizamos las relaciones que existen entre el modelo de evaluación que se lleva a cabo, el Discurso de EF que se defiende y la Racionalidad Curricular en que basamos nuestra práctica docente. Por último, en la tercera parte presentamos una propuesta alternativa, que denominamos «Evaluación Formativay Compartida». Entendemos que esta propuesta posee un mayor valor pedagógico. Hemos comprobado su viabilidad y adecuación, contrastándola en la práctica educativa durante los últimos doce años.

Palabras clave: Evaluación en Educación Física, evaluación formativa, evaluación compartida, discurso de Educación Física, racionalidad y currículum.

\section{Introducción}

Este trabajo pretende ofrecer una alternativa al modelo tradicional de evaluación en Educación Física (EF). Entendemos que existen diez buenas razones para superarle lo antes posible y avanzar hacia modelos de evaluación en nuestra materia con un carácter claramente formativo y educativo. Por eso, en un primer apartado realizamos una crítica al modelo tradicional de evaluación-calificación en EF que, a pesar de las múltiples incoherencias educativas que posee sigue siendo el más utilizado en la práctica educativa y el más enseñado en la formación inicial del profesorado de EF.

A continuación explicamos como el los sistemas de evaluación que ponemos en práctica en Educación Física están fuertemente relacionados con las formas de entender nuestra materia (Enfoques y Discursos de EF), con el modo de utilizar un tipo de Currículum u otro, así como con la Racionalidad Educativa en que se basan nuestros planteamientos teóricos y nuestro conocimiento práctico.

Por último, presentamos la propuesta de evaluación que hemos venido desarrollando y contrastando en los últimos doce años y que ha demostrado ser viable, adecuada y coherente a la hora de avanzar hacia enfoques y prácticas de EF de mayor calidad educativa. Denominamos a esta propuesta: «Evaluación Formativa y Compartida» ${ }^{1}$. Estamos convenidos de que puede ser una forma lógica y coherente de evaluar cuando se están desarrollando metodologías, propuestas, enfoques y/o programas de EF alternativos, superadores del modelo tradicional de EF, enfocado al rendimiento físico deportivo. Si hemos optado por modelos de aprendizaje activo y participativo, que modifican las formas habituales de trabajo entre el alumnado y entre el alumnado y el profesorado, lo lógico es que avancemos también hacía fórmulas colaborativas y formativas de evaluación. 


\section{Análisis crítico del modelo tradicional de evaluación- calificación en Educaicón Física. Razones educativas que hacen urgente su superación}

Cuando hablamos del «modelo tradicional de evaluación en EFì nos estamos refiriendo a la utilización sistemática de test de condición física y/ - habilidad motriz para calificar al alumnado al final de un trimestre o curso en el área de EF. Normalmente, las calificaciones del alumnado se obtiene a partir de sus resultados en dichos test; 0 al menos un porcentaje de las mismas. Aunque sobre este aspecto existe cierta variedad de casos, según los porcentajes aplicados, las tablas normalizadas o los criterios de progresión aplicados.

Entendemos que hay diez grandes razones para dejar de aplicar los habituales test de condición física ( $y / 0$ habilidad motriz) como forma de calificación del alumnado en el área de EF. A continuación vamos a enunciarlas y explicarlas brevemente $^{2}$ :

\subsection{Criticas encontradas en la} bibliografía sobre la utilización de test de condición física como sitema evaluador en EF

En los últimos quince años, diferentes autores han realizado numerosas críticas a la utilización de tests de condición física como sistema de evaluación en EF. Las principales son las siguientes:

En Arnold (1991: 177) podemos encontrar algunas de las deficiencias específicas de los tests referidos a la norma, cuando se utilizan en la escuela.

En Devís y Peiró (1992: 33-35; 1993: 64-66) puede encontrarse una recopilación de las críticas que numerosos autores realizan sobre la utilización de tests de condición física para medir la salud en programas de EF y salud (fundamentalmente centradas en la falta de coherencia entre las finalidades y los instrumentos de evaluación utilizados). Posteriormente Almond (2000) también realiza una crítica a la utilización de test de condición física como sistema de evaluación en los programas de EF y salud; dada su escasa adecuación para la evaluación de la salud física de los individuos. También presenta una serie de propuestas alternativas.
Por su parte, Blázquez (1994: 173-174) recoge seis aspectos críticos sobre la utilización tradicional de los tests como sistema de evaluación en EF. Se trata de una recopilación de aspectos fundamentalmente técnicos, en algunos casos, desde la lógica interna deportiva.

En Méndez (2005: 39) pueden encontrarse las cuatro principales críticas respecto al uso de tests de habilidad para evaluar el rendimiento del juego. Están basadas en los trabajos que realizan algunos de los principales autores del modelo comprensivo de juegos deportivos.

Por nuestra parte, hemos realizado diferentes trabajos donde se critica la patología e incoherencia que supone la utilización de este sistema de evaluación-calificación en nuestra área (López y otros, 1994, 1999, 2006).

\subsection{Se produce un grave reduccionismo de la EF}

Si el modelo de EF que se desarrolla en los centros fuera coherente con este enfoque evaluativocalificativo que se utiliza, la EF se reduciría al entrenamiento de la Condición Física y la ejecución de determinadas Habilidades Motrices. Además, las finalidades de la EF deberían reducirse al logro de objetivos operativos conductuales parcializados y analíticos (de modo que puedan ser objeto de medición), imposibilitando el logro de objetivos más complejos, genéricos y formativos. Dicho de otra manera: la EF debería dedicarse a entrenar cuerpos, no a educar personas en, desde y para los aspectos concerniente al ámbito físicomotriz.

\subsection{Se superficializa el aprendizaje}

Bajo este enfoque sólo se evalúan los objetivos y los contenidos más simples, fáciles y triviales, ya que los test e instrumentos de evaluación denominados «objetivos», y las situaciones de evaluación denominadas «experimentales», sólo miden (¿evalúan?) los niveles de práctica (iaprendizaje?) más básicos y simples. ¿Significa esto que sólo se enseñan los contenidos más simples y analíticos, dado que son los fácilmente medibles de forma «objetiva»?

Las fases más complejas y «ricas» del gesto motor (percepción y decisión) se obvian. Lo mismo ocurre con el resto de características personales, afectivas, sociales y contextuales que se hallan imbricadas. Dada la imposibilidad, o seria dificul- 
tad, de «medirlas cuantitativamente», ni siquiera se tienen en cuenta.

Este tipo de planteamientos y aplicaciones de la «tecnología didáctica» genera un grave problema: es la evaluación la que decide y define los objetivos y contenidos de aprendizaje, en vez de ser al revés, como debería ocurrir en todo proyecto educativo.

\subsection{Su utilización es meramente calificadora}

En la mayoría de los casos, estos sistemas de evaluación se utilizan para calificar numéricamente, de forma impersonal, con aparente asepsia, y bajo falsa presunción de objetividad. No hay ninguna, o muy escasa, intencionalidad educativa ni formativa. Se recurre a ellos cuando se hace necesario emitir una calificación, porque es lo fácil y cómodo; incluso aunque no se corresponda con los objetivos y contenidos desarrollados. También puede recurrirse a ellos porque poseen un aura de neutralidad y solución técnica que evita cualquier tipo de reclamación o implicación personal.

Una vez más, puede observarse la aplicación del mundo del deporte a la EF, puesto que a la hora de la evaluación (calificación) el profesor (entrenador) desempeña funciones de juez deportivo. Es tas funciones son: medir, cronometrar y tabular resultados. Habría que plantearse entonces para qué estudiar cinco años en la universidad, cuando desde el mundo deportivo se pueden formar «jueces» en dos semanas. O bien, si esta función medidora-tabuladora-calificadora no podría ser desempeñada por otra persona cualquiera con una formación mínima, sin alterar en absoluto las condiciones de «objetividad y cientificidad».

\subsection{La adecuación entre la finalidad de la EF y los intrumentos de evaluación utilizados}

Las finalidades más formativas y educativas de la EF, así como las características más valiosas y complejas del movimiento humano, no pueden medirse con los tests físicos y motrices. ¿Qué suele pasar entonces cuando la predominancia de un modelo de evaluación en EF se reproduce curso tras curso y generación tras generación? Quizás, que un porcentaje considerable del profesorado de EF opte por una de las siguientes dos soluciones:
- o se limita a enseñar (entrenar) lo fácilmente medible y registrable,

- o lleva a cabo un diseño curricular más complejo y educativo que no evalúa, pues cuando llega la hora de la calificación se ajusta al modelo dominante de tests de rendimiento físico, concuerde o no con el currículum desarrollado en ese periodo de tiempo.

Ninguna de las dos soluciones parece aceptable, desde un planteamiento didáctico medianamente lógico y responsable.

\subsection{Sobre la acientificidad del proceso de aplicación sobre objetivos complejos}

Una de las críticas más claras que pueden hacerse a muchas de las pruebas y test físicos que se aplican actualmente es su falta de cientificidad al aplicarlos al mundo escolar. Esto es, el incumplimiento de los criterios de verdad y rigor científico por que se rigen (validez interna y externa, fiabilidad y objetividad), incluso con las finalidades de rendimiento y selección deportiva a que dicen servir.

Otro de los aspectos más claramente recogidos, es la reconocida dificultad de «objetivizar» la evaluación de la mayoría de las habilidades motrices y deportivas; lo cual ha conducido al desarrollo de la metodología científica de la observación sistemática, que resulta difícilmente aplicable a las situaciones habituales de trabajo del profesorado de EF.

Probablemente, el mayor problema resida en esa obsesión por la «objetivación», por el cumplimiento de las normas del racionalismo positivista, por querer aplicar a un mundo tan complejo y cambiante como la educación los esquemas de las ciencias naturales.

\subsection{La evalucación y la búsqueda de estatus en EF}

A lo largo de las últimas décadas han ido surgiendo diferentes argumentos que defendían la necesidad de este tipo de enfoques evaluativos para que nuestra asignatura aumentara su estatus académico. Algunos son los siguientes:

A - Por el halo de rigor, cientificidad y objetividad que parecen dar los números, los tests y la cuantificación mecánica. Pero aún en ese caso, los 
sistemas de evaluación predominantes en EF cometen una fuerte y grave incoherencia respecto a las asignaturas de mayor estatus académico; ya que se mide lo que se es, en vez de lo que se aprende sobre los contenidos impartidos.

B - La realización de exámenes teóricos y algunas cuestiones a plantearse. Una de las estrategias desgraciadamente más extendidas ha sido la de dedicar cierto número de sesiones al dictado de apuntes, para posteriormente hacer exámenes «teóricos» de EF.

Plantearemos nuestras críticas a este tipo de prácticas mediante una serie de preguntas:

- chasta que punto se evalúa la comprensión y el conocimiento práctico que esos alumnos poseen sobre algunos contenidos y experiencias, o más bien lo evaluado es su capacidad memorística para retener y reproducir unos conceptos?

- ¿qué contenidos son los que normalmente se «dictan»y se «evalúan» para estos exámenes? ¿A qué modelo de EF pertenecen?

- ¿porqué copiamos las características menos educativas de las otras materias, en vez de hacerlo al revés?

Al igual que Kirk (1990: 78) consideramos que el estatus de la asignatura crecerá por la calidad educativa de su práctica y las convicciones y calidad pedagógica de su profesorado, y no mediante la implantación de exámenes y de contenidos añadidos (conocimiento «científico» biomédico).

En un interesante y reciente trabajo, Velázquez y Hernández (2005: 11-12) analizan cómo las actividades de evaluación de nuestra disciplina acaban perfilando una imagen pedagógica y social de la misma, tanto en el contexto escolar como en el social. Lógicamente, las fuertes contradicciones que existen entre lo que debería ser la EF, y la evaluación predominante del aprendizaje del alumnado que se realiza, no suelen hablar demasiado bien de nuestra materia, ni aportar mucho a su mayor reconocimiento. Estos autores explican cómo muchas de las prácticas habituales de evaluación en EF no responden a los objetivos curriculares y carecen de relevancia educativa. Esto hace que se genere una visión social de la asignatura de bajo prestigio.

\subsection{La disminución del tiempo de enseñanza-aprendizaje}

La mayoría de los procesos tradicionales de evaluación-calificación requieren una considerable cantidad de tiempo para realizarse. Tiempo que se resta del dedicado al proceso de enseñanzaaprendizaje; así como del que pudiera dedicarse a la realización de una evaluación formativa.

En mucha ocasiones hemos podido comprobar cómo el número de sesiones dedicadas específicamente a la evaluación sumativa es algo menor, igual, o incluso superior (según los casos), que el de sesiones dedicadas a actividades de enseñanzaaprendizaje. Existen centros en que un elevado número de las sesiones de cada trimestre se dedican a la realización de este tipo de evaluación, como oportunidad de «recuperación»; y el alumnado que ya ha superado las pruebas, puede dedicarse a la denominada: «actividad libre», «deporte libre», o «jugar un partidillo o pachanguita».

Tanto si analizamos la realidad docente como la literatura especializada, lo que si parece claro es que la evaluación en EF basada en la aplicación de tests y pruebas de rendimiento físico y motriz es la que más tiempo de enseñanza consume. ¿Será precisamente esta otra de las razones de su mantenimiento? ¿Cuál es entonces la cuestión?...

A continuación presentamos algunas posibles:

- ¿Nos sobra tiempo de práctica motriz?

- ¿Es más importante «medir» y comprobar los resultados del alumno, que enseñarle y qué facilitarle experiencias y oportunidades de movimiento y conciencia corporal?

- ¿No es posible realizar la evaluación a través de las actividades cotidianas de enseñanzaaprendizaje, sin tener que dedicar tantas sesiones a este fin específico?

- ¿Nuestra profesión consiste en enseñar y favorecer aprendizajes, o en controlar, medir, registrar, calificar y seleccionar?

\subsection{El desconocimiento y el temor a adentrarse en otros sistemas de evaluación-calificación}

Paralelamente al resto de cuestiones, suele existir cierto temor a adentrarse en otros procesos de evaluación; bien de otros contenidos, bien con diferentes metodologías, supuestamente menos objetivas y medibles; así como involucrarse en procesos evaluativos que suponen un mayor compromiso personal, como la evaluación compartida. En muchos casos dichos temores se deben, fundamentalmente, a una falta de formación o al desconocimiento de recursos para llevarlos a cabo. 


\subsection{Siguiendo el razonamient ¿por qué no medir otras variables?}

Si la evaluación de la EF se reduce a la medición de la condición física, por la dificultad que presenta medir habilidades motrices $y$, dado que las mejoras en el desarrollo de ésta no pueden ser debidas exclusivamente a las sesiones de EF en sus condiciones actuales (estadísticamente hablando),... siguiendo el mismo razonamiento, deberíamos plantearnos por qué no incluir también en la evaluación-calificación la medición antropométrica del alumnado (talla, peso, envergadura, etc...) y su tabulación según las tablas normalizadas de población.

Quizás a través de este razonamiento resulte más obvio que estamos evaluando LO QUE SEES, en vez de LO QUE SE APRENDE. Y una vez en ese punto, deberíamos preguntarnos seriamente... ¿Qué sentido educativo tiene todo esto?

\section{Evaluación, racionalidad y curriculum en Educación Física}

sino que están fuertemente relacionados con modelos, discursos, paradigmas y racionalidades más amplias y complejas, que son las que les dan sentido y coherencia (o se la quitan) en la práctica educativa cotidiana.

En este sentido, el modelo tradicional de evaluación-calificación en EF, que hemos analizado y criticado en el anterior apartado, corresponde de una forma clara y evidente con discursos y enfoques de «EF orientada al rendimiento», así como con un currículum por objetivos y una racionalidad técnica. Por pura coherencia, si nuestros enfoques de EF son diferentes, dicho sistema de evaluacióncalificación no nos vale; al igual que tampoco nos valdría si fuera diferente nuestra racionalidad educativa $y / 0$ nuestra forma de entender y utilizar el currículum.

Cuanto antes tengamos claras dichas relaciones, más fácil será ganar en coherencia, tanto en nuestra práctica como en los modelos teóricos en que apoyamos nuestro trabajo cotidiano.

Vamos a explicar brevemente dichas relaciones, a través de un esquema-resumen (ver tabla I), de forma que puedan comprenderse de forma rápida y clara.

Como puede comprobarse en la Tabla I, el modelo o sistema de evaluación que se elige y se practica está fuertemente relacionado con otros aspectos didácticos más complejos, como el «enfoque curricuTans, el «discurso de Educación Física» y la «racionalidad educativar en que nos basamos. Esto es, la Evaluación forma parte de marcos conceptuales más amplios; no sólo en lo que se refiere al modelo de Educación Física en que se apoya (Discursos de Rendimiento versus Discursos Educativos y de Participación), sino también en el tipo de currículum («Programación por Objetivos» versus «Currículum cómo Proyecto y Proceso»), y de planteamiento educativo (Educación «bancaria», «instructora», «reproductora», «transmisora»... versus Educación «democrática», «comprensiva», «activa», «dialógica»...). ción en Educación Física no constituyen entes aislados ni separados, como a veces puede parecer,

TABLA I. MARCOS DE RACIONALIDAD Y EDUCACIÓN FÍSICA

(Elaboración propia, a partir de López, Monjas y Pérez, 2003)

\begin{tabular}{|c|c|c|}
\hline ASPECTOS & RACIONALIDAD TÉCNICA & RACIONALIDAD PRÁCTICA \\
\hline Interés, Racionalidad & $\begin{array}{l}\text { INSTRUMENTAL, } \\
\text { centrado en los medios }\end{array}$ & $\begin{array}{l}\text { PRÁCTICO, ÉTICO, } \\
\text { centrada en los valores. }\end{array}$ \\
\hline $\begin{array}{l}\text { DISCURSO ED. FÍSICA } \\
\text { (perspectiva, modelo }\end{array}$ & DE RENDIMIENTO & INCLUSIVO, participativo - educativo \\
\hline ENFOQUE CURRICULAR & Currículum por objetivos & Currículum como Proyecto y Proceso \\
\hline $\begin{array}{l}\text { EVALUACIÓN EN } \\
\text { EDUCACIÓN FÍSICA }\end{array}$ & $\begin{array}{l}\text { - La evaluación como medición, } \\
\text { control, calificación y/o poder. } \\
\text { - Obsesión por la "objetividad". } \\
\text { - Utilización de Tests de Condición } \\
\text { Física y habilidad motriz y } \\
\text { deportiva sobre ejecuciones } \\
\text { técnicas concretas. }\end{array}$ & $\begin{array}{l}\text { - Evaluación como diálogo, comprensión y mejora. } \\
\text { - Se da más importancia al proceso que a los resultados. } \\
\text { Preocupación por los procesos de desarrollo personal y grupal. } \\
\text { - Metodología predominantemente cualitativa y enfocada a la } \\
\text { mejora de los aprendizajes y procesos. } \\
\text { - Participación del alumnado en la evaluación. }\end{array}$ \\
\hline
\end{tabular}


Nosotros diferenciamos entre Racionalidad Técnica (o instrumental, o centrada en los medios) y Racionalidad Práctica (o ética, o centrada en los valores).

Cada una de ellas da lugar a planteamientos y prácticas de Evaluación y Enseñanza de la Educación Física marcadamente diferentes, si no opuestas; dado que están basadas en planteamientos ideológicos y profesionales muy distanciados. En concreto, nosotros diferenciamos entre dos grandes concepciones curriculares en la enseñanza de la $E F$; dos grandes formas, opuestas entre sí, de entender y practicar la $\mathrm{EF}^{3}$ : La EF orientada al rendimiento físico-deportivo, y La EF orientada a la educación y la participación (EF. inclusiva).

Esta clasificación está relacionada directamente con las dos concepciones curriculares existentes: el Currículum por objetivos versus el Currículum como Proyecto y Proceso.

\subsection{La necesaria superación de los falsos debates sobre evaluación en EF}

En la mayoría de las publicaciones sobre Evaluación en EF no se entra en las cuestiones que acabamos de comentar, sino que suelen limitarse a los aspectos más superficiales. Por ejemplo: si la evaluación debe ser «objetiva» o «subjetiva», 0 «cuantitativa» o «cualitativa»; o cuando se vuelve a repetir, una y otra vez, la errónea clasificación de "evaluación inicial, formativa y sumativa»; o cuando parece que el único tema a debatir es que porcentaje de calificación hay que aplicar a cada uno de los tipos de contenido ${ }^{4}$. Lo que nosotros entendemos es que, si no se tienen claras las relaciones explicadas entre evaluación, discurso, currículum y racionalidad en EF, nos estaremos limi- tando a reproducir sistemas y modelos de evaluación cuyo sentido y significado ignoramos y que suelen generar fuertes incoherencias en las prácticas educativas cotidianas. Incoherencias que «rechinan» y que suelen dificultar considerablemente el perfeccionamiento profesional.

En la Tabla II explicamos, de forma muy resumida, cómo la diferenciación que aparece en la mayoría de las publicaciones sobre evaluación en EF (evaluación objetiva versus evaluación subjeti$v a$ ), es en realidad un falso debate, tras el que subyacen otros enfrentamiento, debates y dilemas a diferentes niveles de complejidad:

a) a nivel metodológico, entre la metodología cuantitativa vs. la cualitativa;

b) a nivel de formas de entender la Educación Física, entre enfoques de rendimiento vs. de participación;

c) a nivel de concepciones curriculares, entre programación por objetivos vs. currículum como proyecto y proceso;

d) a nivel de marcos de Racionalidad, en que se apoya y adquiere mayor sentido todo lo anterior, entre Racionalidad Técnica vs. Racionalidad Práctica.

\section{Nuestra propuesta de evaluación e Educación Física. La evaluación formativa $y$ compartida. Resultados de su puesta en práctica}

De las diferentes experiencias y procesos de evaluación que hemos generado en estos años, los dos aspectos que consideramos más importantes son:

TABLA II. LOS NIVELES DE DICOTOMÍA DIDÁCTICA EN LA TEMÁTICA

DE LA EVALUACIÓN Y EDUCACIÓN FÍSICA

(Elaboración propia a partir de López et al., 1999, 2006).

\begin{tabular}{|c|c|c|c|}
\hline EVALUACIÓN & OBJETIVA & $\begin{array}{c}\text { aparente } \\
\text { Enfrentamiento }\end{array}$ & SUBJETIVA \\
\hline METODOLOGÍA & CUANTITATIVA & $\begin{array}{c}\text { divergencia } \\
\text { Real }\end{array}$ & $\downarrow \quad$ CUALITATIVA \\
\hline $\begin{array}{l}\text { ENFOQUES DE } \\
\text { EDUCACIÓN FÍSICA }\end{array}$ & $\begin{array}{l}\text { DOMINANTE } \\
\text { (rendimiento) }\end{array}$ & $\begin{array}{c}\text { enfrentamientos } \\
\text { que subyacen }\end{array}$ & $\begin{array}{c}\text { ALTERNATIVOS } \\
\text { (participativo, inclusivo) }\end{array}$ \\
\hline $\begin{array}{l}\text { CONCEPCIÓN } \\
\text { CURRICULAR }\end{array}$ & $\begin{array}{l}\text { CURRÍCULUM POR } \\
\text { OBJETIVOS }\end{array}$ & Basados en las & $\begin{array}{l}\text { CURRÍCULUM COMO } \\
\text { PROYECTO Y PROCESO }\end{array}$ \\
\hline RACIONALIDAD & TÉCNICA & Dicotomías & PRÁCTICA \\
\hline
\end{tabular}


- El esfuerzo por desarrollar una Evaluación eminentemente Formativa en EF.

- El interés por desarrollar procesos e instrumentos que faciliten la realización de una Evaluación Compartida en EF.

Por EVALUACIÓN FORMATIVA entendemos todo proceso de evaluación cuya finalidad principal es mejorar los procesos de enseñanza-aprendizaje que tienen lugar. Es todo proceso de evaluación que sirve para que el alumnado aprenda más (y/o corrija sus errores) y para que el profesorado aprenda a trabajar mejor (a perfeccionar su práctica docente). Por decirlo de otro modo, la finalidad no es calificar al alumno, sino disponer de información que permita saber como ayudar al alumnado a mejorar y aprender más,... y que sirva a su vez para que los profesores aprendamos a hacer nuestro trabajo cada vez mejor.

Para profundizar en nuestras propuestas y experiencias de Evaluación Formativa en EF, aconsejamos consultar los trabajos ya citados.

Por EVALUACIÓN COMPARTIDA entendemos que la evaluación debe ser más un diálogo y una toma de decisiones mutuas y/o colectivas, más que un proceso individual e impuesto. Dentro de estos procesos las autoevaluaciones, las coevaluaciones y las evaluaciones y calificaciones dialogadas son técnicas que juegan un papel fundamental.

La realización de procesos de EVALUACIÓN COMPARTIDA está fundamentada en la búsqueda de una coherencia entre nuestra práctica docente y los planteamientos epistemológicos y pedagógicos en que basamos nuestra teoría y práctica educativa. En este sentido hemos desarrollado una serie de instrumentos y sistemas de evaluación en la Enseñanza de la Educación Física basados en la Evaluación Compartida profesor-alumno. Este sistema ha sido puesto en práctica, estudiado y evaluado en Primaria, Secundaria y Formación del Profesorado (Inicial y Permanente), demostrando su viabilidad y adecuación. Puede encontrarse un detallado desarrollo de estas experiencias en López y otros $(1999,2006)$. Para ver un análisis detallado de las experiencias desarrolladas en nuestro país en este sentido, consultar López Pastor (2004).

\subsection{Realización de una propuesta}

Para dar el difícil salto que relaciona nuestras convicciones didácticas con nuestra práctica docente, lo primero que hicimos fue plantearnos una serie de preguntas-problema a resolver (que constituyen el primer apartado); a continuación elaboramos unos Criterios de Calidad Evaluativa que debían cumplir nuestros sistemas y propuestas de evaluación (segundo apartado); y por último, presentamos algunas líneas de actuación para sistemas e instrumentos concretos de evaluación, a partir del trabajo que hemos desarrollado en los últimos nueve años.

\subsubsection{Las preguntas-problema a resolver}

Las preguntas-problema que intentamos resolver (y que nos ayudan a tomar decisiones sobre los sistemas e instrumentos de evaluación a utilizar) son las siguientes:

a. ¿Cómo integrar la evaluación con los procesos cotidianos de enseñanza-aprendizaje?

b. ¿Cómo estructurar un sistema de evaluación útil para (y al servicio de) la mejora del proceso de enseñanza, el aprendizaje del alumnado y el perfeccionamiento del profesor?

c. ¿Cómo plantear un sistema de evaluación con una orientación claramente formativa y continua?

d. ¿Cómo lograr que sea viable en las condiciones habituales de trabajo de un profesor de EF? ¿Cómo conseguir que sea una ayuda y no una carga?

\subsubsection{Los criterios de calidad educativa}

Como intento de encontrar respuestas a estas preguntas elaboramos una serie de criterios de «Calidad Educativa» que deberíamos intentar cumplir en los sistemas e instrumentos de evaluación que diseñábamos y utilizábamos que son:

-ADECUACIÓN. Se refiere al hecho de que los sistemas e instrumentos de evaluación sean coherentes y adecuados en tres aspectos: respecto al diseño curricular; respecto a las características del alumnado y el contexto; y respecto a los planteamientos docentes.

-RELEVANCIA. Si la información que aporta es relevante, significativa y útil para los implicados en los procesos de enseñanza-aprendizaje.

-VIABILIDAD. Si la utilización sistemática de las técnicas e instrumentos elegidos es viable en las condiciones concretas de trabajo de cada persona.

- VERACIDAD. Si cumplen los criterios de credibilidad y veracidad. ${ }^{5}$ 
- FORMATIVA. En qué grado la evaluación sirve para mejorar el aprendizaje del alumnado, la actuación docente y los procesos de enseñanza-aprendizaje que tienen lugar.

- INTEGRADA. En un doble sentido; en primer lugar si los sistemas e instrumentos de evaluación están integrados en los procesos cotidianos de enseñanza-aprendizaje, en vez de constituir momentos puntuales y finales (este aspecto está relacionado con el concepto de «evaluación continua»-correctamente entendido dicho concepto-); en segundo lugar estar integrados los diferentes sujetos de la evaluación (profesorado, procesos, alumnado) y los diferentes ámbitos y aspectos evaluados.

- ÉTICA. La no utilización de la calificación como herramienta de control, poder, amenaza o venganza sobre el alumnado. Otro aspecto fundamental es el que se refiere a la utilización de la información obtenida a través de la evaluación, en cuanto al cumplimiento de las garantías de confidencialidad y anonimato, así como en cuanto a un uso respetuoso con las personas implicadas. Esto es, en caso de utilizar la información hacerlo en beneficio del alumnado, no en su perjuicio.

\subsubsection{Una propuesta basada en cinco lineas de actuación}

Proponemos trabajar en torno a cinco líneas, instrumentos y dinámicas de evaluación, en las cuales venimos investigando durante los últimos años. Entendemos que estas cinco líneas pueden (y deben) ser complementarias; y en ningún caso excluyentes entre sí.

\section{$A^{\star}$ EL CUADERNO DEL PROFESOR.}

La primera propuesta se basa en la utilización de algún tipo de diario o cuaderno del profesor, donde recoger información sobre los procesos de enseñanza-aprendizaje que van teniendo lugar día a día. Las diferentes posibilidades de esta línea de actuación se mueven entre los dos siguientes extremos:

- cuadernos muy estructurados (que nos ha conducido hacia las «Fichas de autoevaluación» de las sesiones por parte del profesor):

- cuadernos poco o nada estructurados (tendentes a «Anecdotarios, Reflexiones sobre la práctica, Aspectos claves a considerar, Vivencias, diarios personales, etc...).

$B^{\star}$ LAS FICHAS-SESIÓNYLAS FICHASUNIDADES DIDÁCTICAS.

La segunda propuesta se basa en la importancia de diseñar y utilizar apartados (para la observación, el análisis, la reflexión sobre lo acontecido y la toma de decisiones) en las fichas que se utilizan para planificar las Unidades Didácticas (UD.) y las sesiones, de modo que se generen ciclos de reflexión-acción sobre los procesos educativos cotidianos que se llevan a cabo.

Estos procesos de reflexión-acción guardan un fuerte paralelismo con los micro- ciclos de la Investigación-acción, dado que se va pasando de forma recursiva por las fases de: planificación, acción, observación, análisis, reflexión, toma de decisiones (re-planificación),...; generando bucles y espirales de investigación-acción sobre la propia práctica.

C.* PRODUCCIONES DEL ALUMNADO, LA CARPETA YEL CUADERNO DEL ALUMNO.

En este apartado entrarían los diferentes documentos o producciones específicas que realiza el alumnado (de forma individual y/o colectiva) a lo largo de la asignatura. Por ejemplo, fichas de sesiones, trabajos monográficos, proyectos, planes personalizados,...

Cuando el alumnado realiza diferentes documentos a lo largo del curso, una herramienta muy interesante de cara a su acumulación y a la revisión final del proceso, es la carpeta. Una variante de la carpeta, que implica una mayor selección de evidencias y materiales y que, por tanto, puede favorecer aún más la realización de procesos de reflexión sobre el proceso de aprendizaje llevado a cabo, es la técnica conocida como «portafolios».

En esta misma línea, otra opción puede ser la utilización del cuaderno del alumno, si lo entendemos como instrumento de aprendizaje y diálogo profesor-alumno, que además sirve también para hacer una evaluación formativa e integrada. Aunque somos conscientes de que hay que ser extraordinariamente cuidadosos para que no ocurra justo lo contrario de lo que desde aquí planteamos. Esto es, que se utilice el cuaderno para realizar una evaluación fundamentalmente sumativa, con la intención casi exclusiva de calificar.

\section{D* FICHAS Y HOJAS PARA EL ALUMNADO.}

En la inmensa mayoría de los manuales más habituales puede encontrarse una gran diversidad de instrumentos de estas características (listas de control, escalas de observación - numéricas, verbales, gráficas, descriptivas,...-). Nosotros estamos trabajando fundamentalmente en torno a dos grandes tipos de instrumentos que suelen ser muy útiles, tanto para la evaluación del alumnado como para la del proceso y del profesor:

- Fichas de seguimiento individual y hojas de observación grupal. Son fichas de observación, que permiten recoger información sobre los procesos de aprendizaje del alumnado de forma 
sistemática y continua (una unidad didáctica, un trimestre, un curso...).

- Autoinformes, fichas y/o cuestionarios de autoevaluación. Son instrumentos que buscan facilitar la participación del alumnado en los procesos de evaluación, con la intención de mejorar su aprendizaje, así como su responsabilidad y autonomía. Existen muchas posibilidades diferentes, según el contenido, las características del alumnado, el grado de estructuración que se prefiera, la modalidad y tipo de participación, el tiempo y tipo de actividad a evaluar. si son individuales o grupales, etc.

E* DINÁMICAS Y CICLOS DE INVESTIGACIÓNY/O EVALUACIÓN.

En este apartado entran las dinámicas de trabajo colaborativas y grupales dirigidas a la mejora de la propia práctica del profesorado participante, en torno a un tema, o a un problema de la práctica. Habitualmente se siguen dinámicas de Investigación-Acción o Investigación Colaborativa. Para llevar a cabo estos procesos suelen utilizarse diferentes instrumentos y técnicas de obtención de datos, como: Observación externa, $\mathrm{Na}$ rraciones, Descripciones, Grabaciones audio y/o vídeo, Fotografías, Observación sistemática, Informes, etc...

3.2. La importancia de la adecuación de los proceesos e intrumentos de evaluación a las caracteristicas del contexto

Las diferentes experiencias de evaluación que presentamos en nuestros trabajos colectivos, han sido diseñadas y puestas en práctica por profesoras y profesores concretos y en momentos concretos. La diversidad de situaciones profesionales y contextos es tan amplia, que los instrumentos siempre deben ser adaptados de forma específica para cada contexto y/o momento.

En otros trabajos nuestros pueden encontrarse de forma detallada las ventajas e inconvenientes que hemos encontrado según hemos ido poniendo en práctica estas propuestas e instrumentos de evaluación, así como algunas conclusiones finales sobre su viabilidad. Aquí vamos a realizar simplemente una valoración genérica sobre todo el conjunto.

\subsection{Valoración de la propuesta}

La razón de este apartado es que entendemos que es importante resaltar tanto los aspectos posi- tivos y las ventajas como los inconvenientes que conlleva adentrarse por este tipo de procesos evaluadores. En este apartado vamos a presentar un resumen de los principales resultados que hemos obtenido tras su puesta en práctica durante doce años, mediante espirales de investigación-acción. Puede encontrarse una información más detallada de estos procesos y resultados, así como sus análisis de credibilidad, en López y otros $(1999,2006)$.

\subsubsection{Posibles dificultades e inconvenientes que pueden surgir}

Las principales dificultades suelen centrarse en las resistencias que genera su novedad, bien entre alumnado, bien en el resto del profesorado o los padres. Las resistencias del alumnado son las habituales ante cualquier tipo de cambio profundo en sus rutinas y dinámicas de trabajo. En los trabajos desarrollados hemos comprobado que dichas resistencias no suelen durar más allá de uno o dos meses, siempre que el sistema de evaluación esté organizado de un modo coherente y se tengan claras las razones en que se apoya su puesta en práctica.

Un inconveniente a analizar despacio en cada caso, es el relativo al diferente tipo de trabajo que requiere este sistema de evaluación; más continuo y con un enfoque de ayuda y mejora, no de control. Estos cambios tienen dos implicaciones: por un lado son más lentos y costosos, dado que supone remover nuestras costumbres y rutinas más asentadas, así como nuestras algunas de nuestras convicciones; por otro lado, implica una carga de trabajo diferente que, si no se cumple correctamente el criterio de «viabilidad» puede convertirse en una sobrecarga de trabajo que acabe afectando al buen desarrollo de los proceso de evaluación orientados al aprendizaje.

\subsubsection{Ventajas y posibilidades}

En los doce años que llevamos desarrollando esta propuesta, mediante procesos de experimentación e investigación colaborativa, hemos ido comprobando las posibilidades y ventajas que posee, respecto a otros modelos y sistemas de evaluación más tradicionales. Las principales son las siguientes:

- Su indudable valor formativo. Tanto el alumnado como el profesorado implicado nos vamos dando cuenta de las posibilidades formativas que tiene la evaluación, una vez que se supera su habitual identificación con la calificación.

- Su coherencia con nuestras concepciones educativas. Estos sistemas de evaluación han de- 
mostrado ser coherentes con una forma de entender la educación como un proceso colectivo de intercambio y crecimiento mutuo, y no como una mera transmisión de contenidos y/o conocimientos; un planteamiento que posibilita que el alumnado sea protagonista directo y activo del proceso educativo, en especial de la evaluación del mismo en todos sus ámbitos y aspectos.

- Su conexión con procesos de perfeccionamiento profesional. Aunque es cierto que estas líneas de trabajo requieren tiempo y esfuerzo, son una excelente vía de formación permanente del profesorado y perfeccionamiento profesional, dado que implica la evaluación de la propia práctica, con la clara finalidad de irla mejorando; de generar procesos de enseñanzaaprendizaje más ricos y eficaces, así como de ampliar y perfeccionar el conocimiento teórico y práctico del profesorado.

- En los ciclos de investigación-acción desarrollados a lo largo de este tiempo, hemos comprobado en repetidas ocasiones que la propuesta es viable en un alto grado, y que es lo suficientemente flexible para que pueda ser adaptada a todo tipo de contexto y proceso educativo.

- De igual manera, la experiencia acumulada nos ha demostrado que, cuando se ponen en marcha de una forma eficaz este tipo de procesos de evaluación formativa y compartida, suele generar una mejora considerable en tres variables que están estrechamente relacionadas entre si: el aprendizaje del alumnado, su implicación en los procesos de enseñanza-aprendizaje que tienen lugar y el rendimiento académico y los niveles de éxito escolar. $Y$ es un efecto que tiene lugar en todas las etapas del sistema educativo con un sistema acreditativo (primaria, secundaria y universidad).

\section{A modo de conclusión: una invitación a continuar avanzando}

Para finalizar este trabajo, nos gustaría recordar que la colaboración y la búsqueda de nuevas posibilidades a la hora de avanzar hacia una evaluación realmente formativa y educativa es un camino abierto en el que todos estamos invitados y que nos gustaría recorrer juntos. Esperamos que aquellas personas que leáis este trabajo compartáis con nosotros la inquietud por encontrar nuevas respuestas a una temática tan apasionante y cotidiana como es la evaluación educativa.
Confiamos en que algunas de las ideas que aquí se presentan sirvan para continuar avanzando en esta dirección. La mayoría de las personas que las hemos puesto en práctica hemos tenido una experiencia positiva y por eso animamos a que se intente avanzar hacía prácticas más coherentes con nuestras convicciones educativas, a pesar del respeto que suelen generar o de los problemas que pueden surgir durante su aplicación; sobre todo las primeras veces, cuando supone un cambio muy brusco.

\section{Referencias bibliográficas}

ALMOND, L. (2000) «Volviendo a pensar el papel de la salud en Educación Física». En II Congreso Internacional de Educación Física. Jerez de la Frontera. (Cádiz). UGT.

ARNOLD, P. J. (1991) Educación Física, movimiento y currículum. Morata-MEC. Madrid.

BLÁZQUEZ SÁNCHEZ, D. (1994) «Estrategias de evaluación formativa en Educación Física y Deporte». Habilidad Motriz, 4 (5-15).

DEVÍS, J. y PEIRÓ, C. (1992) «Nuevas perspectivas curriculares en Educación Física: la saludy los juegos modificados». Barcelona. Inde.

GUBA, E. (1983) «Criterios de credibilidad en la 01 investigación naturalista». En GIMENO, J. y PÉREZ, A. «La enseñanza: su teoría y su práctica». Madrid. AKAL/Universitaria (148-165).

KIRK, D. (1990). «Educación Física y Currículum». Valencia. Universitat Valencia.

LÓPEZ PASTOR, V. M. (coor.) (1999) «Educación Física, Evaluación y Reforma.. Librería Diagonal. Segovia.

LÓPEZ PASTOR, V. M. (1999) «Prácticas de evaluación en Educación Física: estudio de casos en Primaria, Secundaria y Formación del Profesorado». (Tesis doctoral). Servicio de Publicaciones de la Universidad de Valladolid. Valladolid.

LÓPEZPASTOR, V. M. (2000) «Buscando una evaluación formativa en educación física.». Revista Apunts, $n^{\circ} 62$ (16-26). Ed. INEFC. Barcelona. Madrid

LÓPEZ, V. M. y MONJAS, R. y PÉREZ, D. (2003) Buscando alternativas a la forma de entendery practicar la Educación Física. Ed. INDE. Barcelona.

LÓPEZ PASTOR, V. M. (2004) «La participación del alumnado en los procesos evaluativos: la autoevaluación y la evaluación compartida en educación física». En FRAILE ARANDA, A. «Di- 
dáctica de la Educación Física: una perspectiva crítica y transversal». Ed. Biblioteca Nueva. Madrid. (265-291).

LÓPEZPASTOR, V. M. (coord.) (2006) «La Evaluación en Educación Física: revisión de los modelos tradicionales y planteamiento de una alternativa: la evaluación formativa y compartida». Ed. Miño y Dávila. Buenos Aires.

MÉNDEZ GIMENEZ, A. (2005) «Hacia una evaluación de los aprendizajes consecuente con los modelos alternativos de iniciación deportiva». En Tandem, $n^{\circ} 17$ (38-58). Monográfico sobre la evaluación en Educación Física. Ed. Graó. Barcelona.

PEDRAZA, M., ALONSO, A. y LÓPEZ, V. M. (2002) «La evaluación formativa en primaria: una experiencia con fichas de seguimiento y narrados de sesiones». Revista Aula de Innovación educativa. No115 (24-27). Ed. Graó. Barcelona.

VELÁZQUEZ, R. y HERNÁNDEZ, J. L. (2005) «La Educación Física y su imagen social a la luz de las prácticas de evaluación y calificación del aprendizaje». En Tandem, $n^{\circ} 17$ (7-20). Monográfico sobre la evaluación en Educación Física. Ed. Graó. Barcelona.

VILLAR ÁLVAREZ, F. del (1994) «La credibilidad de la investigación cualitativa en la enseñanza de la Educación Física». Apunts, 37 (26-33). INEFC. Barcelona.

\section{(Notas)}

${ }^{1}$ Pueden encontrarse referencias sobre esta propuesta, así como información de las investigaciones desarrolladas, en anteriores trabajos nuestros (López Pastor y otros, 1999, 2005, 2006; López Pastor, 1999).

2 Para profundizar en este tema es recomendable consultar algunas de las obra anteriormente citadas López, 1999; López y otros, 1999. 2006).

${ }^{3}$ Este planteamiento está basado en los planteamientos y trabajos de autores como: Kirk, 1990; Tinning, 1996: Sparkes, 1992; Fraile, 1995, 1999, 2004; Devís, 1994, 1996; así como en anteriores trabajos nuestros sobre los marcos teóricos y metateóricos en Educación y EF (López Pastor, 1999: López, Monjas y Pérez, 2003).

${ }^{4}$ Hemos analizado estos falsos debates y aparentes dicotomías en anteriores trabajos, así como las ventajas e inconvenientes de la utilización de una u otra metodología de evaluación en EF (López et al., 1999, 2004, 2006).

${ }^{5}$ Para profundizar en estos aspectos consultar: Guba (1983) y del Villar (1994).

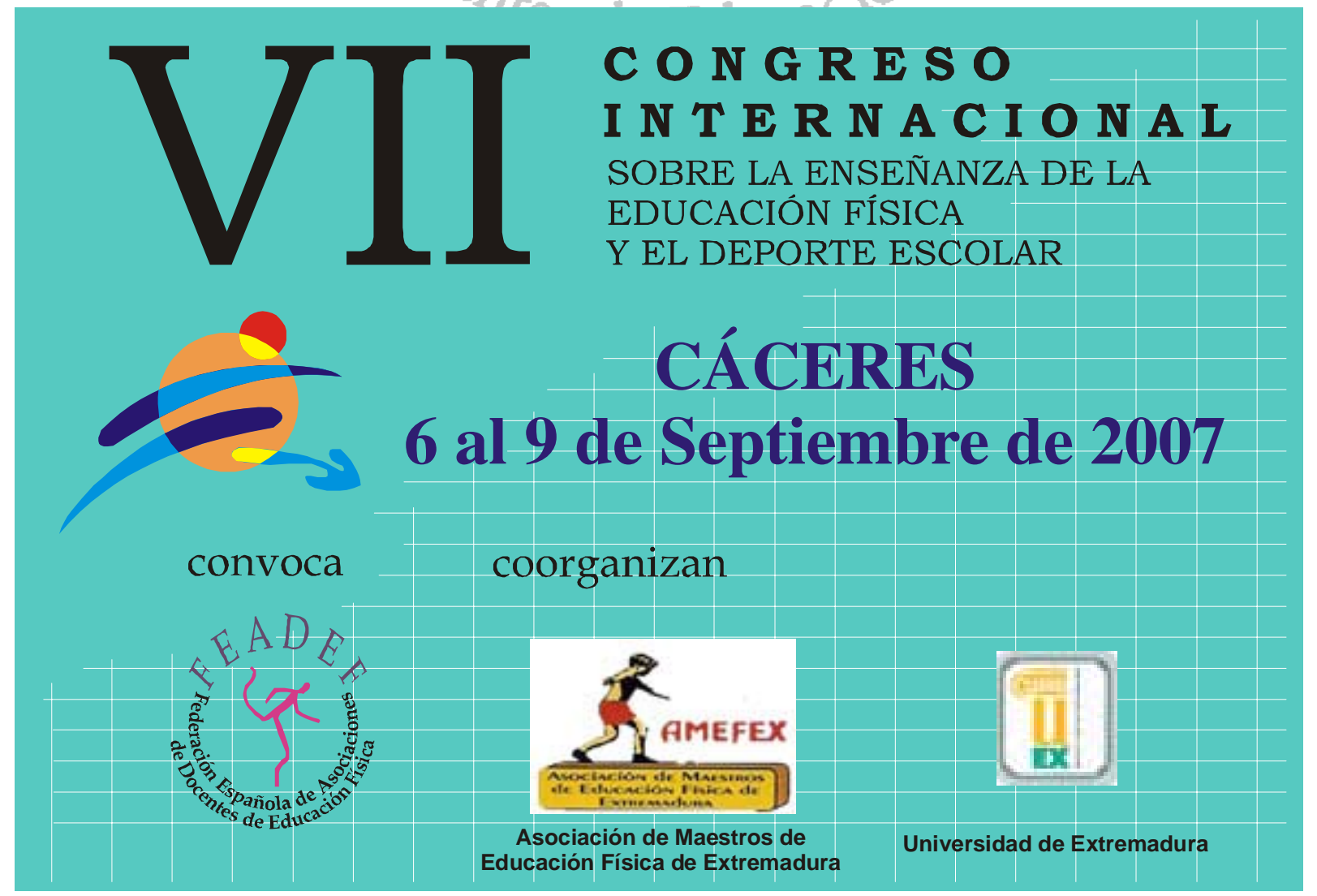

